\title{
Relationship between intracranial internal carotid artery calcification and enlarged cerebral perivascular space
}

\author{
Xiao-Xiao Tao ${ }^{1,2} \cdot{\mathrm{Ge}-\mathrm{Fei} \mathrm{Li}^{1} \cdot \text { Yi-Lan } \mathrm{Wu}^{1} \cdot \mathrm{Yi}^{1} \text {-Sheng Liu }}^{1} \cdot \mathrm{Ying}_{\mathrm{Zhao}}{ }^{1}$. \\ Yan-Hui Shi ${ }^{1}$ - Mei-Ting Zhuang ${ }^{1}$ - Tian-Yu Hou ${ }^{1} \cdot$ Rong Zhao $^{1} \cdot$ Feng-Di Liu $^{1}$ • \\ Xue-Mei Wang ${ }^{1}$ - Ying Shen ${ }^{1}$ - Guo-Hong Cui ${ }^{1}$ - Jing-Jing Su ${ }^{1}$ - Wei Chen ${ }^{1}$. \\ Xue-Mei Tang ${ }^{1,3}$ - Ji Sun ${ }^{1,3}$ • Jian-Ren Liu ${ }^{1,3}$
}

Received: 8 December 2016 / Accepted: 18 April 2017 /Published online: 13 May 2017

(C) The Author(s) 2017. This article is an open access publication

\begin{abstract}
Purpose The association between intracranial internal carotid artery (IICA) calcification and lacunes, white matter hyperintensity (WMH), and cerebral microbleeds (CMBs) has been well researched. However, enlarged cerebral perivascular space (PVS) has not yet been reported to correlate with intracranial internal carotid artery calcification. Therefore, the primary aim of this study was to investigate the relationship between IICA calcification and enlarged PVS.

Methods A total of 189 patients with ischemic stroke in the middle cerebral artery territory who presented within 7 days of ictus from 2012 to 2015 were enrolled respectively. All patients were required to have undergone head computed tomography, magnetic resonance imaging, susceptibility-weighted magnetic resonance imaging, magnetic resonance angiography, or computed tomography angiography. Clinical characteristics were recorded. IICA calcification and enlarged PVS were semi-quantitatively evaluated, and the presence of lacunes, $\mathrm{WMH}$, and CMBs was recorded.
\end{abstract}

$\mathrm{X}-\mathrm{XT}$ and G-FL are joint first authors and contributed equally to this study.

Jian-Ren Liu

liujr021@vip.163.com

1 Department of Neurology, Shanghai Ninth People's Hospital, Shanghai Jiao Tong University School of Medicine, 639 Zhizaoju Road, Shanghai 200011, China

2 Department of Neurology, The First People's Hospital of Wenling, Wenling 317500, China

3 Clinical Research Center, Shanghai Jiao Tong University School of Medicine, Shanghai 200025, People's Republic of China
Results Of the 189 patients, $63.5 \%$ were male. Mean age of the patients was $68.6 \pm 12.2$ years. There were 104 patients with IICA calcification. Age, diabetes mellitus, lacunes, and white matter hyperintensity were significantly associated with IICA calcification $(P<0.05)$. Multivariate logistic regression analysis showed that age, diabetes mellitus, and lacunes were independent predictors of IICA calcification $(P<0.05)$. A lower risk of IICA calcification was found in patients with a higher enlarged PVS score $(P=0.004)$.

Conclusion Higher enlarged PVS scores were associated with a lesser degree of IICA calcification. There appears to be a relationship between reduced risk of IICA calcification and enlarged PVS.

Keywords Intracranial internal carotid artery calcification . Cerebral enlarged perivascular space $\cdot$ PVS $\cdot$ Small vessel disease $\cdot$ Cerebral magnetic resonance imaging $\cdot$ MRI

$\begin{array}{ll}\text { Abbreviations } \\ \text { IICA } & \text { Intracranial internal carotid artery } \\ \text { PVS } & \text { Perivascular spaces } \\ \text { WMH } & \text { White matter hyperintensity } \\ \text { CMB } & \text { Cerebral microbleeds } \\ \text { SVD } & \text { Small vessel disease } \\ \text { MRI } & \text { Magnetic resonance imaging } \\ \text { SWI } & \text { Susceptibility-weighted magnetic resonance images } \\ \text { MRA } & \text { Magnetic resonance angiography } \\ \text { CTA } & \text { Computed tomography angiography } \\ \text { T1WI } & \text { T1-weighted imaging } \\ \text { T2WI } & \text { T2-weighted imaging } \\ \text { DWI } & \text { Diffusion-weighted imaging } \\ \text { FLAIR } & \text { Fluid-attenuated inversion recovery } \\ \text { mIP } & \text { Minimum intensity projection } \\ \text { LAA } & \text { Large artery atherosclerosis }\end{array}$


SVO Small vessel occlusion

CE Cardioembolism

SOE Stroke of other determined etiology

SUE Stroke of undetermined etiology

\section{Introduction}

Intracranial internal carotid artery (IICA) calcification is the most common type of intracranial arterial calcification $[1,2]$. With the use of the Woodcock Visual Scoring system [3] and semiautomatic calculation of calcium score [4] as well as improvement in cranial computed tomography (CT) and computed tomography angiography (CTA), calcification of the IICA has been a focus in many studies. Currently, the relationship between IICA calcification and carotid atherosclerosis, incidence of stroke, and etiology of stroke is still controversial, and the mechanism underlying IICA calcification is still poorly understood.

Perivascular spaces (PVS), which are also called VirchowRobin spaces (VRS), are normal anatomic structures in the nervous system [5]. They are formed when perforating arteries and outflow veins take the pia mater with them when they dive deep into the brain. Studies have confirmed that the VirchowRobin spaces of capillary beds are occluded, and the interstitial fluid is static in the central nervous system [6]. The enlarged PVS have been found to be closely related to some pathological processes of the brain such as the atrophy of brain parenchyma and alteration of vascular permeability $[7,8]$. On the basis of microscopic structural difference in pathophysiology, enlarged PVS can be mainly divided into two types. One is the basal ganglia type, in which the lenticulostriate arteries project into the basal ganglia via the anterior perforating arterial branch. The enlarged PVS of basal ganglia type has been confirmed to be closely related to cerebral small vessel disease (SVD). Basal ganglia-enlarged PVS have been reported to be associated with older age, centrum semiovale-enlarged PVS, cerebral atrophy, and lacunar stroke subtype [9, 10]; basal ganglia-enlarged PVS have also been associated with cognitive impairment [11]. The other type of enlarged PVS is the cerebral hemisphere type, in which medullary arteries enter the convex gray matter of the cerebral hemisphere and then extend to the subcortical white matter (centrum semiovale); thus, it is also known as cerebral white matter type. Neuroimaging studies have revealed that enlarged PVS, together with status lacunaris, white matter signal hyperintensity, and cerebral microbleeds, have been found to be markers related to cerebral SVD [9, 12, 13]. These four markers have been integrated as a system that is used to evaluate SVD (overall SVD scoring system) [14], which have been widely applied in clinical practice for the systemic evaluation of cerebral SVD.
In recent years, arterial calcification has been regarded as a discernible, highly specific marker of atherosclerosis in imaging. Coronary artery calcification has been confirmed to be related to coronary atherosclerotic plaques and can be used to predict the occurrence of coronary arterial events such as myocardial infarction and angina $[15,16]$. There is evidence showing that the incidence of intracranial carotid calcification in stroke patients is significantly higher than in non-stroke subjects [2].

Many studies have shown that intracranial carotid calcification is closely related to status lacunaris, white matter signal hyperintensity, and microbleeds [17-21]. However, little is known about the relationship between intracranial carotid calcification and enlarged PVS as a kind of marker of cerebral SVD. Therefore, the primary aim of this study was to investigate the relationship between intracranial carotid calcification and enlarged PVS.

\section{Materials and method}

\section{Patients}

This was a retrospective study. The study included 189 patients diagnosed with ischemic stroke due to obstruction of the middle cerebral artery (MCA) at the Department of Neurology and Jiuyuan Municipal Stroke Center of Shanghai Ninth People's Hospital, Shanghai Jiao Tong University School of Medicine between June 2012 and December 2015.

\section{Patient recruitment}

The inclusion criteria were the following: (1) received treatments in the Department of Neurology of Shanghai Ninth People's Hospital, Shanghai Jiao Tong University School of Medicine between June 2012 and December 2015; (2) had a first ischemic stroke, older than 40 years, unilateral obstruction of the MCA, and no hemorrhagic transformation (cranial $\mathrm{CT}$ showed hyperintensity at infarct area or cranial susceptibility-weighted magnetic resonance imaging (SWI) showed heterogeneous intensity); (3) underwent cranial plain MRI, cranial SWI, cranial plain CT, cranial magnetic resonance angiography (MRA), or cranial CT angiography. The interval between cranial plain MRI and cranial CT was no longer than 6 months; and (4) the images obtained were clear.

The exclusion criteria were the following: (1) evident malacia lesions in brain parenchyma, old infarction, hemorrhage, or tumor; (2) severe brain atrophy or hydrocephalus; (3) received thrombolytic therapy or revascularization (including arterial embolectomy and arterial stenting) before admission; (4) obvious artifacts in the images due to some reasons (such as denture); and (5) MRI parameters were adjusted due to special requirements. 


\section{Clinical information at baseline}

Age, gender, hypertension, diabetes mellitus (DM), hyperlipidemia, atrial fibrillation, coronary heart disease, and smoking status were recorded as risk factors of cerebrovascular diseases. Hypertension was defined as mean systolic blood pressure $(\mathrm{SBP})>140 \mathrm{mmHg}$ and/or mean diastolic blood pressure (DBP) $>90 \mathrm{mmHg}$ at two consecutive non-invasive measurements with an interval of at least $15 \mathrm{~min}$, or a medical history of oral medication with blood pressure lowering drugs (such as diuretics, calcium channel blockers, angiotensin-converting enzyme inhibitors, etc.). Diabetes mellitus was defined as fasting plasma glucose $>7.9 \mathrm{mmol} / \mathrm{L}$, plasma glucose at any time of $>11.0 \mathrm{mmol} / \mathrm{L}$, or a history of oral medication of glucose lowering drugs. Hyperlipidemia was defined as lowdensity lipoprotein $>160 \mathrm{mg} / \mathrm{dL}$, total cholesterol of $>240 \mathrm{mg} /$ $\mathrm{dL}$, triglycerides of $>150 \mathrm{mg} / \mathrm{dL}$, or a history of oral medication with lipid-lowering drugs. Coronary heart disease was defined as the presence of clinical manifestations of myocardial infarction, angina, or ischemic heart failure as well as coronary atherosclerosis. Smoking was defined as smoking $>10$ cigarettes per day or smoking for $>1$ year.

\section{Neuroimaging findings}

All the patients underwent MRI scanning with a 3.0T MR scanner (Siemens, Germany), and images were obtained after axial scanning.

Cranial CT was performed with a 64-slice dual-source spiral CT (Siemens, Germany) (slice thickness 5 or $10 \mathrm{~mm}$; FOV: $512 \times 512)$.

Cranial MRI was performed with the following parameters: slice thickness: $5 \mathrm{~mm}$; slice interval: $6.5 \mathrm{~mm}$; T1WI sequence: TR, $1800 \mathrm{~ms}$; TE, $8.8 \mathrm{~ms}$, FOV, $640 \times 576$; T2WI sequence: TR, $4500 \mathrm{~ms}$; TE, $106 \mathrm{~ms}$; FOV, $640 \times 576$; FLAIR sequence: TR, $7000 \mathrm{~ms}$; TE, $89 \mathrm{~ms}$; FOV, $512 \times 464$; DWI sequence: TR, 4000 ms; TE: 100 ms; FOV, $288 \times 324$.

Susceptibility-weighted magnetic resonance imaging was performed with high-resolution, three-dimensional gradientecho (3D-GE) sequence as follows: magnitude image (Mag): TR, $28.0 \mathrm{~ms}$; TE, $20.0 \mathrm{~ms}$; slice thickness, $1.5 \mathrm{~mm}$; FOV, $640 \times 520$; phase image (Pha): TR, $28.0 \mathrm{~ms}$; TE, $20.0 \mathrm{~ms}$; slice thickness, $1.5 \mathrm{~mm}$; FOV, $640 \times 520$; minimum intensity projection (mIP): TR, $28.0 \mathrm{~ms}$; TE, $20.0 \mathrm{~ms}$; slice thickness, $12.0 \mathrm{~mm}$; FOV, $640 \times 520$; SWI image: TR, $28.0 \mathrm{~ms}$; TE, $20.0 \mathrm{~ms}$; slice thickness, $1.5 \mathrm{~mm}$; FOV, $640 \times 520$.

\section{Neuroimaging analysis}

The images on the PACS screen were evaluated by a neurologist and radiologist who were blind to the clinical characteristics and prognosis of these patients and received standardized training. The same template was used for determination of location, and degree of calcification, white matter hypersensitivity, status lacunaris, and microbleed were evaluated. Cerebral magnetic resonance angiography was performed with the following parameters: TR, $22 \mathrm{~ms}$; TE, $3.6 \mathrm{~ms}$; flip angle: $20 \mathrm{o}$; slice thickness, $1.5 \mathrm{~mm}$; slice interval, $1.8 \mathrm{~mm}$; FOV, $640 \times 580$. Computed tomography angiography was performed with a 64-slice dual-source spiral CT (Siemens, Germany). Gadolinium solution was injected at $4.5 \mathrm{~mL} / \mathrm{s}$. The slice thickness was $1 \mathrm{~mm}$; the FOV was $512 \times 512$.

\section{Degree of calcification}

The bilateral intracranial carotid siphon was used as region of interest for the evaluation of degree of calcification. The consistent window (length $800 \mathrm{HU}$; width $2000 \mathrm{HU}$ ) was used in cranial plain $\mathrm{CT}$, and the bilateral intracranial carotid siphon was evaluated with the Woodcock assessment system [3], with the highest degree recorded. Calcification was graded as 1 , mild (thin and discontinuous calcification); 2, intermediate (thin and continuous calcification), thick or discontinuous calcification; 3 , severe (thick and continuous calcification) (Fig. 1).

A. Semi-quantitative assessment of region of interest of the intracranial carotid artery: From petrous segment to internal carotid artery, bifurcation was performed.

B. Evaluation of calcification of the intracranial carotid artery was based on the following classification: grade 1 (thin and discontinuous calcification), grade 2 (thin and continuous calcification, or thick and discontinuous calcification), grade 3 (thick and continuous calcification).

\section{Semi-quantitative assessment of perivascular space}

The extent of PVS dilation was semi-quantitatively evaluated with the criteria developed by MacLullich et al. [22]: 0 , no PVS; 1, <10 PVS; 2, 11-20 PVS; 3, 21-40 PVS; 4, >40 PVS. Counting of PVS was done in the hemisphere with the largest number. Figure 2 shows the location of the basal ganglia. Of note, macroscopic observation is more sensitive to hyperintensity than to hypointensity. In the present study, the PVS was counted on the T2WI sequence. Figure 3 shows the PVS of $1-4$.

\section{Assessment of white matter hyperintensity}

The criteria of Fazekas et al. [23] were employed to evaluate the deep white matter and white matter next to the lateral ventricle. Hyperintensity of white matter next to the lateral ventricle: 0 , no lesion; 1 , cat-like or pencil-like thin lesions; 2 , lesion with smooth halo; 3 , irregular hyperintensity next to the lateral ventricle extending to deep white matter. 


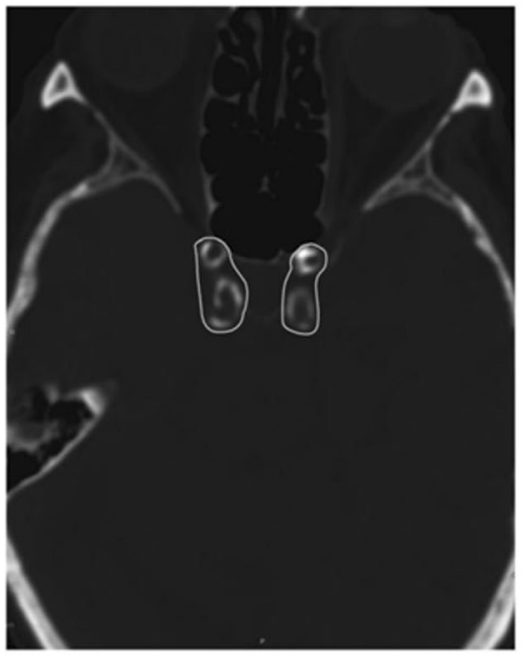

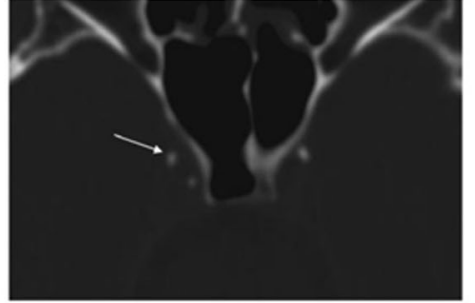

Grade 1: Thin, discontinuous calcifications

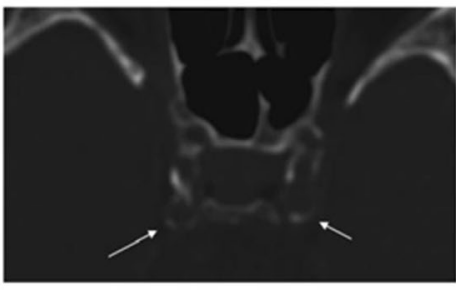

Grade 2: Thick, discontinuous calcifications

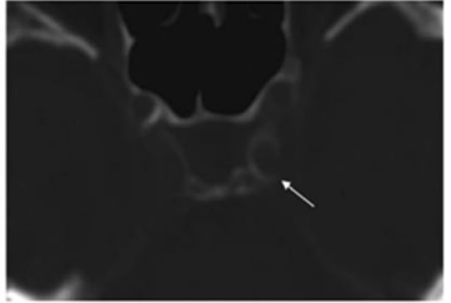

Grade 2: Thin, continuous calcifications

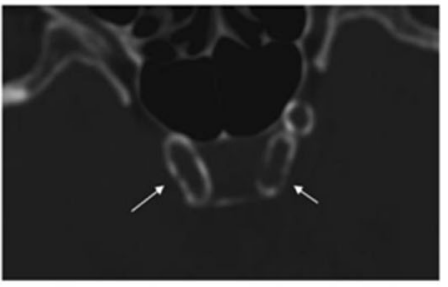

Grade 3: Thick, continuous calcifications

Fig. 1 The area of interest for evaluating the degree of intracranial internal carotid artery calcification and examples for three grades

Hyperintensity of deep white matter: 0 , no lesion; 1 , spotty lesions; 2 , lesions merged; 3 , massive merged lesions.

The hyperintensity of white matter was defined as a score 3 for hyperintensity of white matter next to the lateral ventricle and/or score 2-3 of hyperintensity of deep white matter (Fig. 4). The presence of white matter hyperintensity was recorded. Of note, the white matter hyperintensity secondary to acute brain infarction was not included for evaluation.

\section{Assessment of status lacunaris}

Status lacunaris, also known as lacunar infarction, and characterized by the round or oval lesions (3-20 $\mathrm{mm}$ in diameter) in the basal ganglia, internal capsule, centrum ovale, or brainstem which show similar intensity as cerebral spinal fluid on T2WI or T2 fluid-attenuated inversion recovery (FLAIR). Status lacunaris is hard to differentiate from enlarged PVS on T2WI and is mainly differentiated by FLAIR. Generally, on

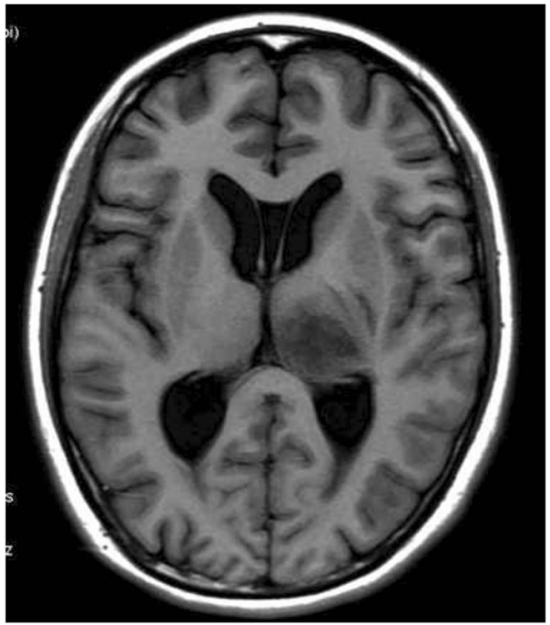

Fig. 2 Axial cranial MRI localization slice at the level of the basal ganglia
FLAIR, the lesions of lacunar infarction show hyperintensity at the periphery and hypointensity at the center; meanwhile, they have no hyperintensity on DWI [24] (Fig. 5). In this study, only the number of lacunar infarction lesions at the basal ganglia was counted.

\section{Evaluation of microbleeds}

Microbleeds are defined as round or oval lesions $(2-10 \mathrm{~mm}$ in diameter) with hypointensity on T2WI or SWI. Generally, they are not observable on cranial CT, FLAIR, T1WI, or T2WI. These lesions may be present in the cerebellum, brainstem, basal ganglia, white matter, and cortex-subcortex junction (21). Microbleeds are defined as one or more microhemorragic lesions in the brain parenchyma (Fig. 6). The presence of microbleeds was recorded. Microbleed lesions are also common in vascular amyloidosis (43) which is also a SVD. Thus, vascular amyloidosis was not excluded.

\section{TOAST subtyping}

According to the subtyping criteria of the Trial of Org 10172 in Acute Stroke Treatment (TOAST) [19], the etiology of stroke was subtyped in each patient as follows: large artery atherosclerosis (LAA), small vessel occlusion (SVO), cardioembolism (CE), stroke of other determined etiology (SOE), and stroke of undetermined etiology (SUE).

\section{Statistical analysis}

Mean and standard deviation were computed for age, and frequency and percentage were calculated for all categorical variables. To compare the difference between patients with and without IICA calcification, chi-square test or Fisher's exact test for categorical variables and independent $t$ test for age 
Fig. 3 Example of different extent of enlarged perivascular space scoring from 1 to 4 at the level of the basal ganglia. a PVS for 1 point, $\mathbf{b}$ PVS for 2 points, $\mathbf{c}$ PVS for 3 points, $\mathbf{d}$ PVS for 4 points

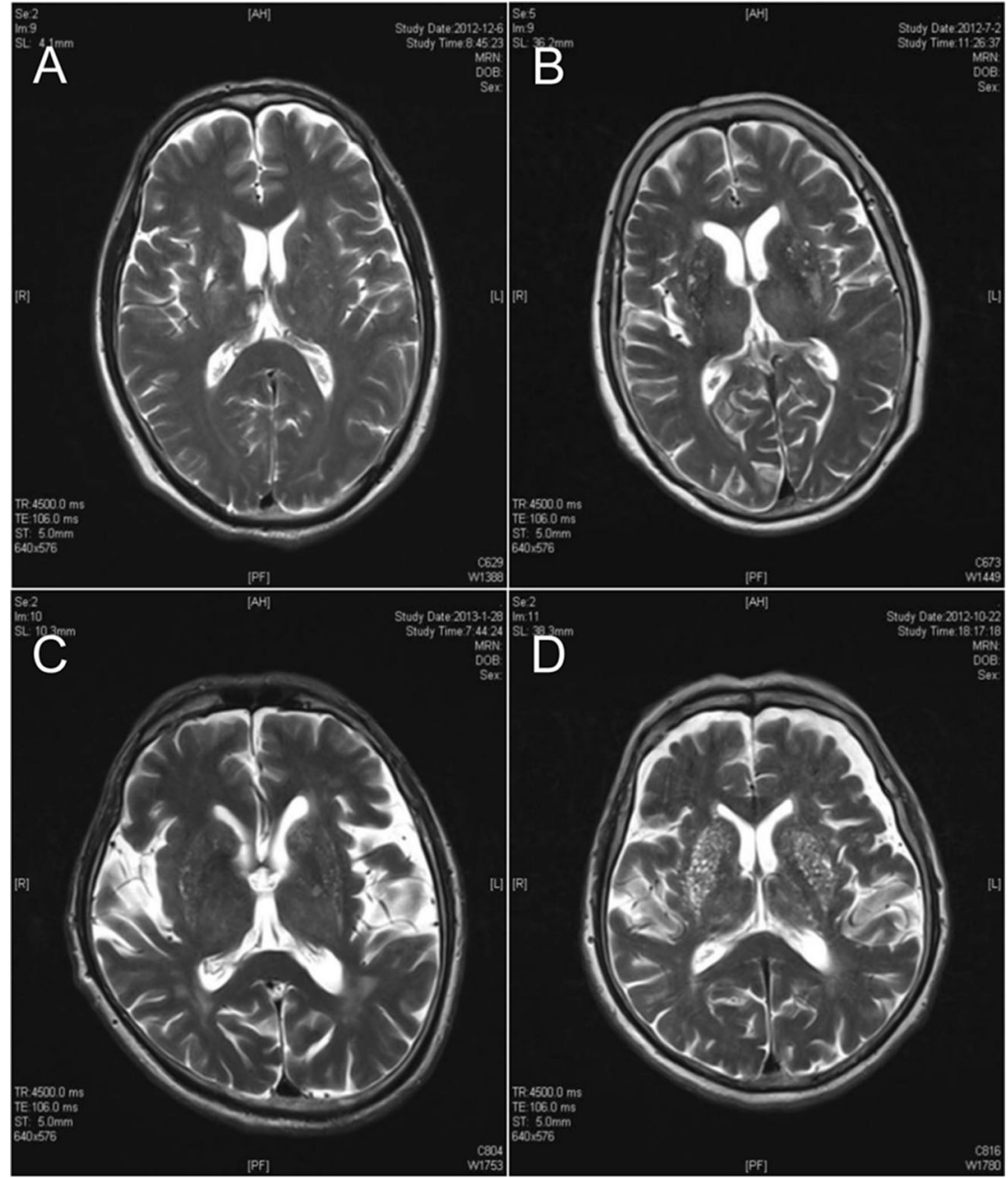

was used. Multivariate binary logistic regression analysis was implemented to determine the risk factors of IICA calcification based on the results of univariate analysis. Meanwhile, ordinal logistic regression was carried to examine the risk factors of severity of IICA calcification. Significance level was set at 0.05 for all analyses. All data were analyzed with PASW 22 (IBM Corp., Armonk, NY, USA).

\section{Results}

Table 1 shows the characteristics of the patients with and without IICA calcification. There were some similarities between the two groups such as gender, smoking, hypertension, cardiovascular disease, TOAST classification, and cerebral microbleeds, but patients with IICA calcification tended to
Fig. 4 Example of

periventricular and deep white matter hyperintensity. a High signal of lateral ventricle white matter with Fazekas 3 points (which elongate to deep white matter). b High signal in deep white matter with Fazekas 2 points (pathological changes of fusion)

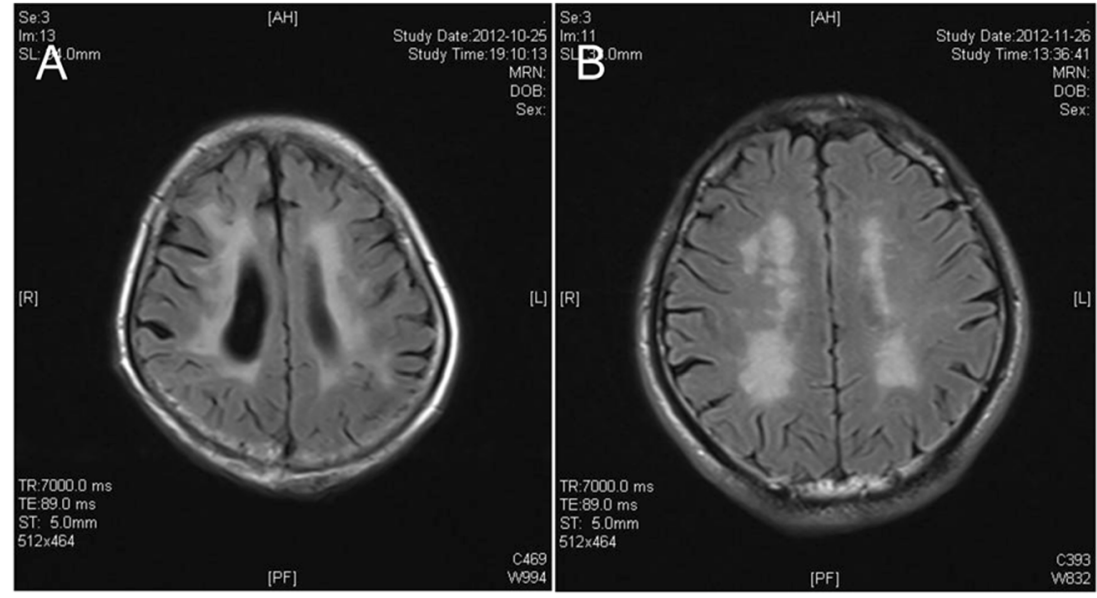


Fig. 5 a Axial cranial T2 weighted MRI shows a lacunar infarct lesion of hyperintensity (white arrow). b Axial cranial FLAIR shows a lacunar infarct lesion with ring of hyperintensity and core of hypointensity (white arrow)

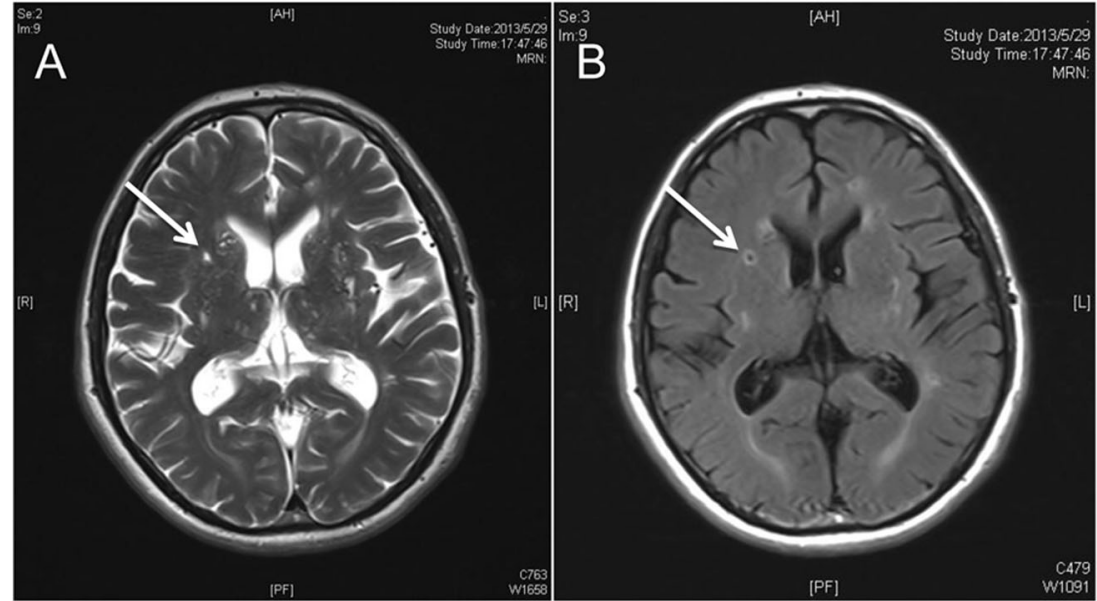

be older (72.9 vs. 63.4 years, $P<0.001)$, more prone to diabetes ( 39.4 vs. $17.6 \%, P=0.001)$, and having 0 -point enlarged PVS score ( 31.7 vs. $16.5 \%, P=0.047)$, lacunes (68.3 vs. $36.5 \%, P<0.001$ ), and white matter hyperintensity (36.5 vs. $16.5 \%, P=0.002)$. The final model revealed that advanced age $(\mathrm{OR}=1.08,95 \%$ CI $1.05-1.12, P<0.001)$ was related to higher risk of IICA calcification. In addition, patients with diabetes or lacunes had about threefold $(\mathrm{OR}=3.03,95 \% \mathrm{CI}$ 1.37-6.71, $P=0.006)$ or fourfold (OR $=4.24,95 \%$ CI 2.04 $8.80, P<0.001)$ higher risk of IICA calcification compared with those without diabetes or lacunes. Conversely, a lower risk of IICA calcification was found in patients with 1-point $(\mathrm{OR}=0.37,95 \%$ CI $0.05-0.91, P=0.030)$ or higher enlarged PVS score $(\mathrm{OR}=0.17,95 \%$ CI $0.06-0.48, P<0.001)$ (Table 2).

Table 3 summarizes risk factors of severity of IICA calcification. In univariate analysis, age was found to be related to IICA calcification deterioration, and male gender was related to a minor severity; however, only the effect of age was significant in the multivariate analysis $(\mathrm{OR}=1.04,95 \% \mathrm{CI}$ $1.01-1.08, P=0.021)$.

\section{Discussion}

In this study, which is the first to investigate the association between IICA calcification and enlarged cerebral PVS, it was found that enlarged PVS were associated with a lower risk of IICA calcification. Multivariate logistic regression analysis showed that age was an independent risk factor for increased severity of IICA calcification. This finding is consistent with a previously reported finding that calcification volume in IICA calcification is positively associated with age [25].

In previous studies on coronary arterial calcification and calcification of common carotid bifurcation, the incidence of calcification was higher and the calcification volume was larger in males [26-28]. This may be related to the higher prevalence of smoking, drinking, and hyperlipidemia in males than
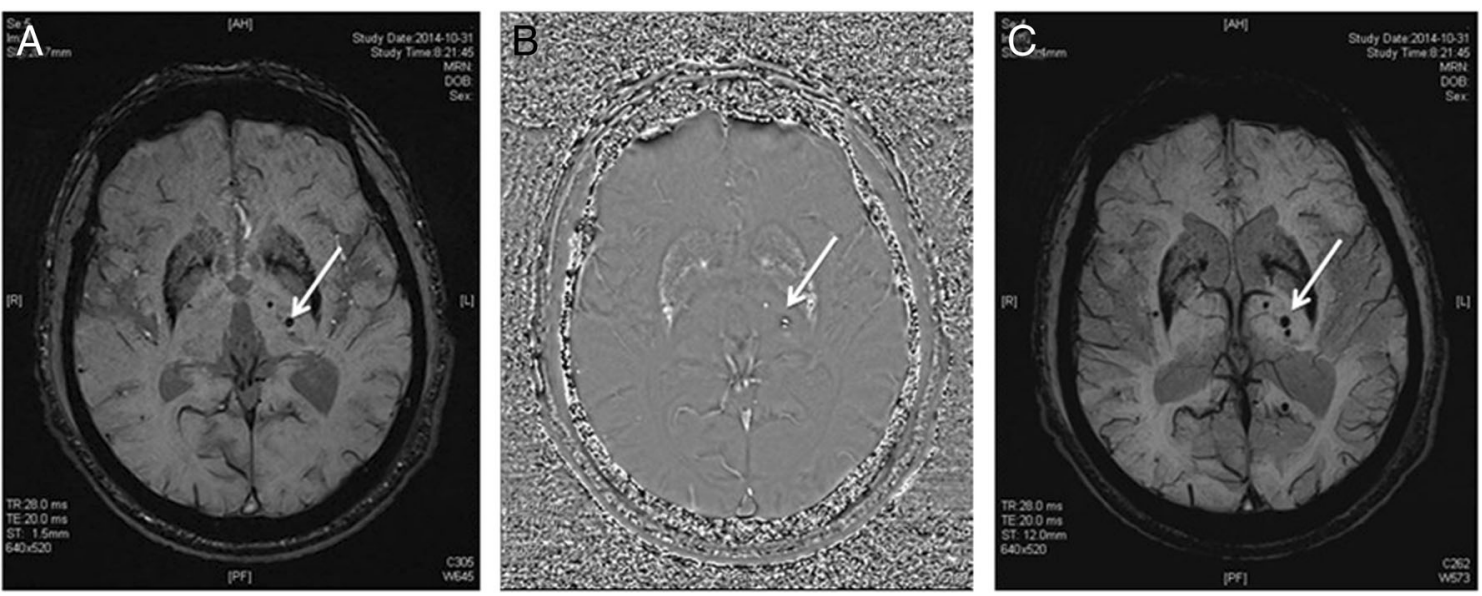

Fig. 6 Different signals of cerebral microbleeds (CMBs) on sequences of SWI Phase and mIP. a SWI sequence: 2 lesions of microbleed on the left basal ganglia which are expressed as low signal (white arrow). b Phase sequence: 2 microbleed lesions on the left basal ganglia which are expressed as high signals (white arrow). c mIP sequence: 2 microbleed lesions on the left basal ganglia which are expressed as low signals (white arrow) 
Table 1 Clinical characteristics of 189 patients

\begin{tabular}{|c|c|c|c|}
\hline & \multicolumn{3}{|c|}{ Intracranial internal carotid artery calcification } \\
\hline & No $(n=85)$ & Yes $(n=104)$ & $P$ \\
\hline Age, years & $63.4 \pm 11.9$ & $72.9 \pm 10.8$ & $<0.001$ \\
\hline Males & $58(68.2)$ & $62(59.6)$ & 0.221 \\
\hline Smoking & $38(44.7)$ & $37(35.6)$ & 0.202 \\
\hline Hypertension & $58(68.2)$ & $82(78.8)$ & 0.098 \\
\hline Diabetes & $15(17.6)$ & $41(39.4)$ & 0.001 \\
\hline Hyperlipidemia & $7(8.2)$ & $3(2.9)$ & 0.102 \\
\hline Cardiovascular disease & $6(7.1)$ & $16(15.4)$ & 0.076 \\
\hline TOAST & & & 0.506 \\
\hline Large artery atherosclerosis & $14(16.5)$ & $20(19.2)$ & \\
\hline Small vessel occlusion & $42(49.4)$ & $51(49)$ & \\
\hline Cardioembolism & $3(3.5)$ & $8(7.7)$ & \\
\hline Undetermined etiology & $26(30.6)$ & $25(24)$ & \\
\hline Enlarged PVS score & & & 0.047 \\
\hline 0 & $14(16.5)$ & $33(31.7)$ & \\
\hline 1 & $44(51.8)$ & $41(39.4)$ & \\
\hline $2-4$ & $27(31.7)$ & $30(28.9)$ & \\
\hline Scanning slice thickness, $\mathrm{mm}$ & & & 0.002 \\
\hline 5 & $27(31.8)$ & $56(53.8)$ & \\
\hline 10 & $58(68.2)$ & $48(46.2)$ & \\
\hline Lacunes & $31(36.5)$ & $71(68.3)$ & $<0.001$ \\
\hline Cerebral microbleeds & $25(29.4)$ & $38(36.5)$ & 0.301 \\
\hline White matter hyperintensity & $14(16.5)$ & $38(36.5)$ & 0.002 \\
\hline
\end{tabular}

Data on age are presented as mean \pm standard deviation and others are expressed as number $(\%)$.

Italicized values indicate statistically significant $P<0.05$

PVS perivascular space, TOAST Trial of Org 10172 in Acute Stroke Treatment in females as these are risk factors for cerebrovascular diseases. Our results showed that male gender was negatively related to calcification, and there was significant difference

Table 2 Risk factors for intracranial internal carotid artery calcification $(n=189)$

\begin{tabular}{|c|c|c|}
\hline & Odds ratio ( $95 \%$ confidence interval) & $P$ \\
\hline Age, years & $1.08(1.05,1.12)$ & $<0.001$ \\
\hline \multicolumn{3}{|l|}{ Diabetes } \\
\hline No & Reference & \\
\hline Yes & $3.03(1.37,6.71)$ & 0.006 \\
\hline \multicolumn{3}{|c|}{ Score of enlarged perivascular space } \\
\hline 0 & Reference & \\
\hline 1 & $0.37(0.15,0.91)$ & 0.030 \\
\hline $2-4$ & $0.17(0.06,0.48)$ & $<0.001$ \\
\hline \multicolumn{3}{|l|}{ Lacunes } \\
\hline No & Reference & \\
\hline Yes & $4.24(2.04,8.80)$ & $<0.001$ \\
\hline
\end{tabular}

Forward logistic regression was performed.

Italicized values indicate statistically significant, $P<0.05$. in gender among patients with different degrees of calcification, and more patients with mild calcification were males. However, the sample size was relatively small, and ischemic stroke patients were included. Thus, our findings require further confirmation.

There is evidence showing that DM is not only an independent risk factor for intracranial carotid calcification $[29,30]$ but is closely related to calcification of the extracranial carotid artery, coronary artery, and aortic arch [31, 32]. Yilmaz et al. [32] also found that DM was independently related to the calcification loading of intracranial carotid calcification. In this study, $\mathrm{DM}$, age, and status lacunaris as risk factors of cerebrovascular diseases were included in multivariate logistic regression analysis, and the results also showed DM was independently associated with intracranial carotid calcification $(P<0.05)$. Diabetes mellitus may cause high inflammatory state, abnormal lipid metabolism, and abnormal activity of the fibrinolytic system and has been regarded as an important factor involved in the initiation and progression of atherosclerosis [33]. Our findings may be helpful for the elucidation of the relationship between intracranial carotid calcification and atherosclerosis and provide evidence for future studies in this field. 
Table 3 Risk factors for severity of intracranial internal carotid artery calcification in 104 patients

\begin{tabular}{|c|c|c|c|c|}
\hline & \multicolumn{2}{|l|}{ Univariate } & \multicolumn{2}{|l|}{ Multivariable } \\
\hline & OR $(95 \% \mathrm{CI})$ & $P$ & OR $(95 \% \mathrm{CI})$ & $P$ \\
\hline Age, years & $1.06(1.02,1.1)$ & 0.003 & $1.04(1.01,1.08)$ & 0.021 \\
\hline Males & $0.34(0.16,0.75)$ & 0.007 & $0.46(0.2,1.03)$ & 0.060 \\
\hline Smoking & $0.57(0.26,1.24)$ & 0.158 & & \\
\hline Hypertension & $0.81(0.33,1.99)$ & 0.653 & & \\
\hline Diabetes & $0.94(0.44,1.97)$ & 0.861 & & \\
\hline Hyperlipidemia & $8.69(0.75,100.92)$ & 0.084 & & \\
\hline Cardiovascular disease & $1.47(0.53,4.07)$ & 0.454 & & \\
\hline \multicolumn{5}{|l|}{ TOAST } \\
\hline Large artery atherosclerosis & Reference & & & \\
\hline Small vessel occlusion & $0.85(0.41,0.57)$ & 0.665 & & \\
\hline Cardioembolism & $1.98(0.57,0.15)$ & 0.281 & & \\
\hline Undetermined etiology & $0.8(0.36,0.56)$ & 0.595 & & \\
\hline \multicolumn{5}{|l|}{ Enlarged PVS score } \\
\hline 0 & Reference & & & \\
\hline 1 & $0.55(0.28,0.95)$ & 0.073 & & \\
\hline $2-4$ & $0.73(0.36,0.68)$ & 0.382 & & \\
\hline Lacunes & $0.7(0.32,1.54)$ & 0.373 & & \\
\hline Cerebral microbleeds & $1.14(0.53,2.42)$ & 0.744 & & \\
\hline White matter hyperintensity & $1.15(0.54,2.46)$ & 0.718 & & \\
\hline
\end{tabular}

Italicized values indicate statistically significant $P<0.05$

$C I$ confidence interval, $O R$ odds ratio, PVS perivascular space, TOAST Trial of Org 10,172 in Acute Stroke Treatment
Enlarged PVS (EPVS) were identified on T2WI and MPRAGE T1 and defined as round, ovoid, or linear structures with CSF-like signal, no larger than $3 \mathrm{~mm}$ in diameter and located in territories supplied by perforating arteries. EPVS in both basal ganglia and centrum semiovale regions were rated according to a previously published 4-point semi-quantitative score $[9,34]$. We assessed EPVS in the basal ganglia and centrum semiovale separately because it was possible that due to their different locations and features, they could have distinct pathophysiology. EPVS in both the basal ganglia and centrum semiovale were coded with the following scale applied to standard axial images: 0 indicating no EPVS, 1 indicating 10 EPVS, 2 indicating 11 to 20 EPVS, 3 indicating 21 to 40 EPVS, and 4 indicating more than 40 EPVS. The numbers referred to PVS on one side of the brain; the higher score was used if there was asymmetry between the sides and EPVS were counted in the slice with the highest number.

Our results showed the grade of enlarged PVS was similar between patients with and without calcification and in those with different degrees of calcification. However, multivariate logistic regression analysis showed enlarged PVS was associated with a reduced risk for IICA calcification $(P=0.004)$, i.e., the higher the enlarged PVS score, the milder the calcification. The non-significant difference in the score of enlarged PVS might be ascribed to small number of patients with enlarged
PVS of grade 3-4 and the abnormal distribution of enlarged PVS of different grades.

In the present cross-sectional study, it was not possible to clarify the cause-effect relationship between increased enlarged PVSs and reduced risk of intracranial internal carotid artery calcification. The causal relationship should be confirmed in prospective studies with long-term follow-up. Our findings suggest that enlarged PVS are different from other cerebral SVDs, such as status lacunaris, white matter signal hyperintensity, and microbleeds, that are positively related to cerebrovascular calcification [17-21] and that the pathogenic mechanism of enlarged PVS is different from that of cerebrovascular calcification. This provides evidence for the need to further explore the mechanism underlying the pathogenesis of enlarged PVS.

It should also be noted that basal ganglia-enlarged PVS have been associated with the lacunar stroke subtype [9, 10], deep and periventricular white matter hyperintensity [9], and cognitive impairment. On the other hand, centrum semiovaleenlarged PVS were not found to be associated with any particular ischemic stroke subtype, deep or periventricular white matter hyperintensity [9], or cognitive impairment [35]. That is why we only analyzed the association between basal ganglia enlarged PVS and intracranial internal carotid artery calcification. 
Our study had several limitations. This was a retrospective study. Also, the sample size was small due to time and recruitment limitations. Thus, all the risk factors related to intracranial carotid calcification were not covered in this study, and future prospective studies with large sample size are required to confirm our findings. In addition, there might be selection bias in the patients' recruitment because patients with severe stroke are unable to undergo cranial MRI and other imaging examinations. Thus, the results obtained in this study might be biased.

\section{Conclusion}

Our results showed basal ganglia-enlarged PVS are associated with a reduced risk of IICA calcification, i.e., the higher the grade of PVS, the milder the calcification. This is the first study to investigate the relationship between PVS dilation and IICA calcification. In addition, our results also showed age, DM, and status lacunaris were independent predictors of IICA calcification, which is consistent with previous reports.

\section{Compliance with ethical standards}

Funding This work was funded by the National Natural Science Foundation of China (81271302 to JRL), the Shanghai Jiao Tong University Medical Engineering Cross Research Foundation (no. YG2014MS07 to JJS), the Research Innovation Project from the Shanghai Municipal Science and Technology Commission (no. 14JC1404300 to JRL), the "Prevention and Control of Chronic Diseases Project" of Shanghai Hospital Development Center (no. SHDC12015310 to JRL), the SHSMU-ION Research Center for Brain Disorders (no. 2015NKX006 to JRL), the Shanghai Municipal Education Commission - Gaofeng Clinical Medicine Grant Support (no. 20161422 to JRL), the Clinical Research Project from Shanghai Jiao Tong University School of Medicine (no. DLY201614 to JRL) and the Biomedicine Key Program from the Shanghai Municipal Science and Technology Commission (no. 16411953100 to JRL).

Conflict of interest The authors declare that they have no conflict of interest.

Ethical approval All procedures performed in the studies involving human participants were in accordance with the ethical standards of the institutional and/or national research committee and with the 1964 Helsinki Declaration and its later amendments or comparable ethical standards.

Informed consent Informed consent was obtained from all individual participants included in the study.

Open Access This article is distributed under the terms of the Creative Commons Attribution 4.0 International License (http:// creativecommons.org/licenses/by/4.0/), which permits unrestricted use, distribution, and reproduction in any medium, provided you give appropriate credit to the original author(s) and the source, provide a link to the Creative Commons license, and indicate if changes were made.

\section{References}

1. Chiewvit P, Tritrakam SO, Kraumak T (2015) Computed tomography evaluation of intracranial vascular calcification in major ischemic stroke patients (vascular territory) - its distribution and association with vascular risk factors: a retrospective trial. J Med Assoc Thail 98:414-422

2. Chen XY, Lam WW, Ng HK, Fan YH, Wong KS (2007) Intracranial artery calcification: a newly identified risk factor of ischemic stroke. J Neuroimaging 17:300-303

3. Woodcock RJ Jr, Goldstein JH, Kallmes DF, Cloft HJ, Phillips CD (1999) Angiographic correlation of CT calcification in the carotid siphon. AJNR Am J Neuroradiol 20:495-499

4. Subedi D, Zishan US, Chappel F et al (2015) Intracranial carotid calicificationon cranial computed tomography: visual scoring methods, semiautomated scores, and volume measurements in patients with stroke. J Stroke 46:2504-2509

5. Braffman BH, Zimmerman RA, Trojanowski JQ, Gonatas NK, Hickey WF, Schlaepfer WW (1988) Brain MR: pathologic correlation with gross and histopathology. 1. Lacunar infarction and Virchow-Robin spaces. AJR Am J Roentgenol 9:551-558

6. Brinker T, Stopa E, Morrison J, Klinge P (2014) A new look at cerebrospinal fluid circulation. Fluids Barriers CNS 11:10. doi:10. 1186/2045-8118-11-10 eCollection 2014

7. Yao M, Hervé D, Jouvent E et al (2014) Dilated perivascular spaces in small-vessel disease: a study in CADASIL. Cerebrovasc Dis 37: $155-163$

8. Wuerfel J, Haertle M, Waiczies H et al (2008) Perivascular spaces - MRI marker of inflammatory activity in the brain? Brain 131:2332-2340

9. Doubal FN, MacLullich AM, Ferguson KJ, Dennis MS, Wardlaw JM (2010) Enlarged perivascular spaces on MRI are a feature of cerebral small vessel disease. Stroke 41:450-454

10. Potter GM, Doubal FN, Jackson CA et al (2015) Enlarged perivascular spaces and cerebral small vessel disease. Int J Stroke 10:376-381

11. Arba F, Quinn TJ, Hankey GJ et al (2016) Enlarged perivascular spaces and cognitive impairment after stroke and transient ischemic attack. Int J Stroke Aug 19. Pii: 1747493016666091

12. Wardlaw J, Smith C, Dichgans M (2013) Mechanisms of sporadic cerebral small vessel disease: insights from neuroimaging. Lancet Neurol 12:483-497

13. Rouhl RP, Van Oostenbrugge RJ, Knottnerus IL, Staals JE, Lodder J (2008) Virchow-Robin spaces relate to cerebral small vessel disease severity. J Neurol 255:692-696

14. Staals J, Makin SD, Doubal FN, Dennis MS, Wardlaw JM (2014) Stroke subtype, vascular risk factors, and total MRI brain smallvessel disease burden. Neurology 83:1228-1234

15. O'Leary DH, Polak JF, Kronmal RA, Manolio TA, Burke GL, Wolfson SK Jr (1999) Carotid-artery intima and media thickness as a risk factor for myocardial infarction and stroke in older adults. Cardiovascular Health Study Collaborative Research Group. N Engl J Med 340:14-22

16. Sung JH, Yeboah J, Lee JE et al (2016) Diagnostic value of cornary artery calcium score for cardiovascular disease in African Americans: the Jackson Heart Study. Br J Med Med Res 11:1-9

17. Chung PW, Park KY, Kim JM, Shin DW, Ha SY (2014) Carotid artery calcification is associated with deep cerebral microbleeds. Eur Neurol 72:60-63

18. Hong NR, Sen HS, Lee YH et al (2011) The correlation between carotid siphon calcification and lacunar infarction. Neuroradiology 53:643-649

19. Del Brutto OH, Mera RM, Gillman J, Ha JE, Zambrano M (2015) Calcifications in the carotid siphon correlate with silent cerebral small vessel disease in community-dwelling older adults: a 
population-based study in rural Ecuador. Geriatr GerontolInt. doi: 10.1111/ggi.12599

20. Chung PW, Park KY, Moon HS (2010) Intracranial internal carotid artery calcification: a representative for cerebral artery calcification and association with white matter hyperintensities. Cerebrovasc Dis 30:65-71

21. Bos D, Ikram MA, Elias-Smale SE (2011) Calcification in major vessel beds relates to vascular brain disease. Arterioscler Thromb Vasc Biol 31:2331-2337

22. MacLullich AM, Wardlaw JM, Ferguson KJ et al (2004) Enlarged perivascular spaces are associated with cognitive function in healthy elderly men. J Neurol Neurosurg Psychiatry 75:1519-1523

23. Fazekas F, Chawluk JB, Alavi A et al (1987) MR signal abnormalities at $1.5 \mathrm{~T}$ in Alzheimer's dementia and normal aging. AJR Am J Roentgenol 149:351-356

24. Wardlaw JM, Smith EE, Biessels GJ et al (2013) Neuroimaging standards for research into small vessel disease and its contribution to ageing and neurodegeneration. Lancet Neurol 12:822-838

25. Knudsen KA, Rosand J, Karluk D, Greenberg SM (2001) Clinical diagnosis of cerebral amyloid angiopathy: validation of the Boston criteria. Neurology 56:537-539

26. deWeert TT, Cakir H, Rozie S et al (2009) Intracranial internal carotid artery calcifications: association with vascular risk factors and ischemic cerebrovascular disease. AJNR Am J Neuroradiol 30: 177-184

27. Janowitz WR, Agatston AS, Kaplan G, Viamonte M Jr (1993) Differences in prevalence and extent of coronary artery calcium detected by ultrafast computed tomography in asymptomatic men and women. Am J Cardiol 72:247-254

28. Odink AE, van der Lugt A, Hofman A et al (2007) Association between calcification in the coronary arteries, aortic arch and carotid arteries: the Rotterdam study. Atherosclerosis 193:408-413

29. Lopez-Cancio E, Dorado L, Millan M et al (2012) The BarcelonaAsymptomatic Intracranial Atherosclerosis (AsIA) study: prevalence and risk factors. Atherosclerosis 221:221-225

30. Mazighi M, Labreuche J, Gongora-Rivera F, Duyckaerts C, Hauw JJ, Amarenco P (2008) Autopsy prevalence of intracranial atherosclerosis inpatients with fatal stroke. Stroke 39:1142-1147

31. Odink AE, van der Lugt A, Hofman A et al (2010) Risk factors for coronary, aortic arch and carotid calcification; the Rotterdam Study. J Hum Hypertens 24:86-92

32. Yilmaz A, Akpinar E, Topcuoglu MA (2015) Clinical and imaging features associated with intracranial internal carotid artery calcifications in patients with ischemic stroke. Neuroradiology 57:501506

33. Kadoglou NP, Avgerinos ED, Liapis CD (2010) An update on markers of carotid atherosclerosis in patients with type 2 diabetes. Biomark Med 4:601-609

34. Pantoni L (2010) Cerebral small vessel disease: from pathogenesis and clinical characteristics to therapeutic challenges. Lancet Neurol 9:689-701

35. Huijts M, Duits A, Staals J, Kroon AA, de Leeuw PW, van Oostenbrugge RJ (2014) Basal ganglia enlarged perivascular spaces are linked to cognitive function in patients with cerebral small vessel disease. Curr Neurovasc Res 11:136-141 